\title{
Encorafenib-Binimetinib-Cetuximab Regimen
}

National Cancer Institute

\section{Source}

National Cancer Institute. Encorafenib-Binimetinib-Cetuximab Regimen. NCI Thesaurus. Code C161595.

A reg imen consisting of encorafenib, binimetinib and cetuximab that can be used in the treatment of certain BRAF-mutated colon and rectal cancers. 